\title{
Multiple-Enzyme-Digestion Strategy Improves Accuracy and Sensitivity of Label- and Standard-Free Absolute Quantification to a Level That Is Achievable by Analysis with Stable Isotope-Labeled Standard Spiking
}

\author{
Jacek R Wiśniewski, ${ }^{* \dagger \odot ~ C h r i s t i n e ~ W e g l e r, ~}{ }^{\ddagger},{ }^{\circ}$ and Per Artursson ${ }^{\ddagger}, \|$ \\ ${ }^{\dagger}$ Biochemical Proteomics Group, Department of Proteomics and Signal Transduction, Max-Planck-Institute of Biochemistry, Am \\ Klopferspitz 18, D-82152 Martinsried, Germany \\ ${ }^{*}$ Department of Pharmacy, Uppsala University, S-751 23 Uppsala, Sweden \\ ${ }^{\S}$ Cardiovascular, Renal and Metabolism, Innovative Medicines and Early Development Biotech Unit, AstraZeneca, Gothenburg, \\ Sweden \\ "Science for Life Laboratory, Uppsala University, S-751 23, Uppsala, Sweden
}

Supporting Information

\begin{abstract}
Quantification of individual proteins is an essential task in understanding biological processes. For example, determination of concentrations of proteins transporting and metabolizing xenobiotics is a prerequisite for drug disposition predictions in humans based on in vitro data. So far, this task has frequently been accomplished by targeted proteomics. This type of analyses requires preparation of stable isotope labeled standards for each protein of interest. The selection of appropriate standard peptides is usually tedious and the number of proteins that can be studied in a single experiment by these approaches is limited. In addition, incomplete digestion of proteins often affects the accuracy of

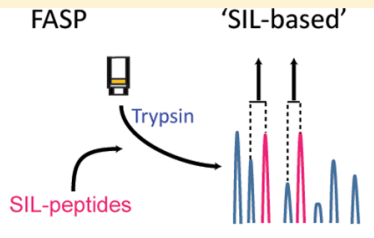

MED-FASP
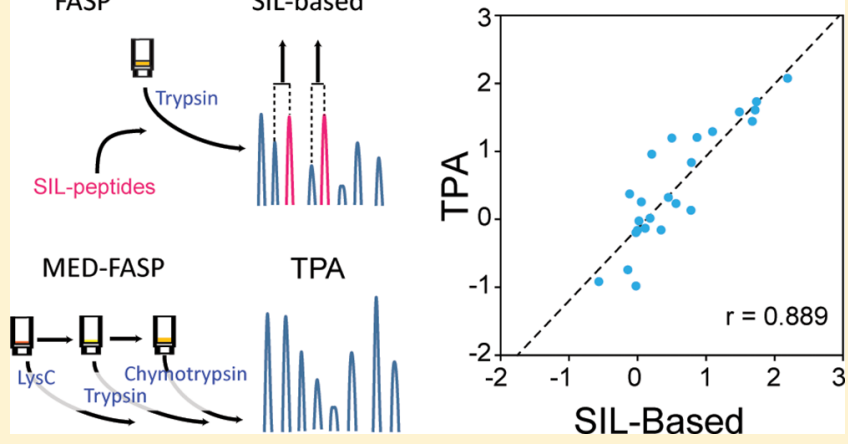
proteomic protein quantification, label- and standard-free approaches, such as "total protein approach" (TPA) have been proposed. Here we directly compare an approach using stable isotope labeled (SIL) standards and TPA for quantification of transporters and enzymes in human liver samples within the same LC-MS/MS runs. We show that TPA is a convenient alternative to SIL-based methods. Optimization of the sample preparation beyond commonly used single tryptic digestion, by adding consecutive cleavage steps, improves accuracy and reproducibility of the TPA method to a level, which is achievable by analysis using stable isotope-labeled standard spiking.
\end{abstract}

KEYWORDS: FASP, MED FASP, total protein approach (TPA), stable isotope labeling, quantitative analysis, drug transporter, drug metabolizing enzymes, $A D M E$

\section{INTRODUCTION}

Information on concentrations of individual proteins is a prerequisite for understanding biological systems. In classical biochemistry this information is commonly achieved by immunoassays or staining techniques. Alternatively, abundance of proteins or enzymes can be assessed indirectly by studying ligand binding or measuring rates of substrate conversion by enzymes. With the advent of mass spectrometry based proteomics, other techniques for determination of protein concentrations became popular: so-called "targeted analysis" allows quantification of individual peptides by comparing spectral intensities of their ions and the ion of stable isotope labeled peptide standards. Despite the continuously increasing popularity of this approach, the targeted analysis-similar to the biochemical approaches-is limited to a preselected and usually small number of proteins quantifiable at the same time in a single experiment. In addition, the biochemical and the targeted proteomic analyses share common biases such as inaccuracies in determination of standard and total protein content. Provided it is properly validated, targeted analysis may be useful for rapid assessment of single or smaller numbers of proteins, but it is missing the omics perspective. The fundamental mission of proteomics is to procure global information on individual proteins encompassed in a system. By quantifying as many proteins as possible, functions in biological and physiological systems can more readily be elucidated, for instance by pathway analysis. The total protein

Received: July 17, 2018

Published: October 18, 2018 
approach (TPA $)^{1-3}$ combined with the proteomic ruler ${ }^{4}$ offer quantification of thousands of proteins across data sets, providing protein concentrations and protein copy numbers. Importantly, in contrast to other label free methods, this approach directly provides values with physicochemical units, without any standards or accompanying assays such as total protein contents determination or cell counting. In the past we have demonstrated the convenience of using TPA by analyses of, for example, bacterial, ${ }^{2}$ animal, ${ }^{5,6}$ and human systems. ${ }^{7-9}$ Notably, several recent publications from other laboratories stresses a convenience and accuracy of our approach for data interpretation, e.g. ${ }^{10-13}$

Determination of protein concentrations of drug transporters and drug metabolizing enzymes are of importance for predicting drug disposition in humans based on in vitro data. This task is most frequently completed by proteomic analyses using SIL peptide standards. In this work, we compare in the same analytical run a SIL-based analysis with a global proteomic approach using TPA quantification. Further, we demonstrate that the accuracy of TPA quantification can be significantly improved by using consecutive protein digestion with two or three enzymes.

\section{EXPERIMENTAL SECTION}

\section{Human Tissue}

Normal liver tissue samples were obtained from patients undergoing liver resection at Uppsala University Hospital (Uppsala, Sweden) (Uppsala Regional Ethical approval no. 2009/028). All donors gave their informed consent. The nontumorous liver tissue samples were snap-frozen upon resection and stored at $-150{ }^{\circ} \mathrm{C}$ until further proteomic analysis.

\section{Labeled Peptides}

Important drug metabolizing enzymes and drug transporters were quantified in this study. Proteotypic peptides of the proteins were chosen based on the procedure used by Gröer et al., ${ }^{14}$ which combines in silico predictions and experimental data. Peptides obtained from in silico trypsin digestion (https://web.expasy.org/peptide_cutter/) had a defined sequence length of 7-25 amino acids and excluded any missed cleavages sites. Peptides containing cysteine, methionine, tryptophan or $\mathrm{N}$-terminal glutamic acid, transmembrane region, or regions where post-translational modifications, polymorphisms or mutations could occur, were excluded. Finally, protein specificity of each peptide was determined using UniProtKB/Swiss-Prot. Peptides with isotopically labeled lysine $\left({ }^{13} \mathrm{C} 6,{ }^{15} \mathrm{~N} 2\right)$ and arginine $\left({ }^{13} \mathrm{C} 6,{ }^{15} \mathrm{~N} 4\right)$ residues were purchased from JPT Peptide Technology and New England Peptide. Selected peptides were verified in tryptic digests using a QTRAP6500 (Sciex), ensuring that the correct MS/MS-transitions could be determined at the same retention times, for each peptide, as has been described previously. ${ }^{14}$

\section{Preparation of Tissue Lysates}

Thawed pieces (about $50 \mathrm{mg}$ ) of human liver tissue were homogenized on ice in $2 \%$ SDS in $0.1 \mathrm{M}$ Tris- $\mathrm{HCl}, \mathrm{pH} 8.0$, containing $0.1 \mathrm{M} \mathrm{DTT}$ and lysed as described previously. ${ }^{8}$ Total protein was determined using WF-assay in microtiter plate format. $^{15}$
Multienzyme Digestion Filter Aided Sample Preparation (MED FASP)

Sample aliquots containing $50 \mu \mathrm{g}$ total protein were processed using either $\mathrm{FASP}^{16}$ or MED FASP ${ }^{17}$ method, with some modifications. ${ }^{18}$ For the stable isotope labeled (SIL)-based analysis, proteins were sequentially digested with LysC and trypsin, whereas for the TPA based quantification the lysates were consecutively cleaved with LysC, trypsin and chymotrypsin, as described recently. ${ }^{19}$ Aliquots of tryptic digests, containing $10 \mu \mathrm{g}$ of total peptide, were spiked with $0.25,0.5,1$, or $2 \mathrm{pmol}$ of the labeled peptides (Supporting Information (SI) Table 1). Samples were analyzed in triplicates.

Liquid Chromatography-Tandem Mass Spectrometry

Analysis of peptide mixtures was performed using a QExactive HF-X mass spectrometer (Thermo-Fisher Scientific, Palo Alto). Aliquots containing $1 \mu \mathrm{g}$ of total peptide were chromatographed on a $50 \mathrm{~cm}$ column with $75 \mu \mathrm{m}$ inner diameter packed $\mathrm{C}_{18}$ material. Peptide separation was carried out at $300 \mathrm{~nL} / \mathrm{min}$ for $95 \mathrm{~min}$ using an acetonitrile gradient of $5-30 \%$. The temperature of the column oven was $55{ }^{\circ} \mathrm{C}$. The mass spectrometer operated in data-dependent mode with survey scans acquired at a resolution of 60000 . Up to the top 15 most abundant isotope patterns with charge $\geq+2$ from the survey scan $(300-1650 \mathrm{~m} / z)$ were selected with an isolation window of $1.4 \mathrm{~m} / z$ and fragmented by HCD with normalized collision energies of 25 . The maximum ion injection times for the survey scan and the MS/MS scans were 20 and $28 \mathrm{~ms}$, respectively. The ion target value for MS1 and MS2 scan modes was set to $3 \times 10^{6}$ and $10^{5}$, respectively. The dynamic exclusion was $30 \mathrm{~s}$. The mass spectrometry data have been deposited to the ProteomeXchange Consortium via the PRIDE partner repository ${ }^{20}$ with the data set identifier: PXD009482.

Data Analysis

The MS spectra were searched using MaxQuant software. A maximum of two missed cleavages was allowed. The maximum false peptide and protein discovery rate was specified as 0.01 . The concentration of the proteins were calculated from ratios of $\mathrm{MS}^{1}$ spectral intensities of "light" (analyte) to "heavy" (stable isotope labeled spiked-in standard) peptides as reported by the MaxQuant software using the relationship:

$$
c(i)=\frac{L}{H} \times \frac{C(i)_{\mathrm{STD}(i)}}{m}
$$

Where $\frac{L}{H}$ is the ratio of "light" to "heavy" peptides; $c(i)_{\operatorname{STD}(i)}$, molar amount of spiked-in labeled standard; $m$, total mass of peptides.

The TPA methods was used for calculation of protein concentrations of all proteins. ${ }^{21}$ The calculations were performed in Microsoft Excel using the relationship:

$$
c(i)=\frac{\mathrm{MS}-\operatorname{signal}(i)}{\text { TotalMS-signal } \times \mathrm{MW}(i)}\left[\frac{\mathrm{mol}}{\text { gtotalprotein }}\right]
$$

Where MS-signal (i) refers to the sum of $\mathrm{MS}^{1}$ spectral intensities of all peptides matching sequence of protein $i$ and Total MS-signal is the sum of $\mathrm{MS}^{1}$-spectral intensities of all identified peptides. 


\section{A. Protein quantification by 'targeted' \& TPA analysis}

FASP: Succesive digestion

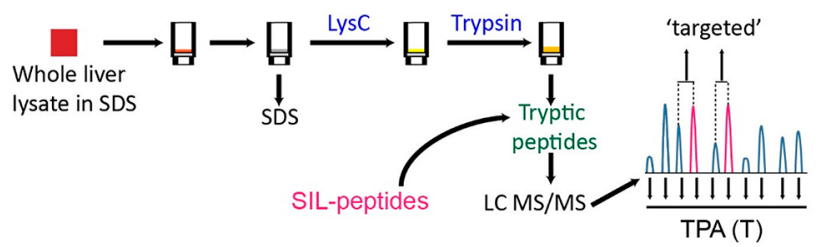

B. Protein quantification by TPA analysis

MED-FASP: Consecutive digestion

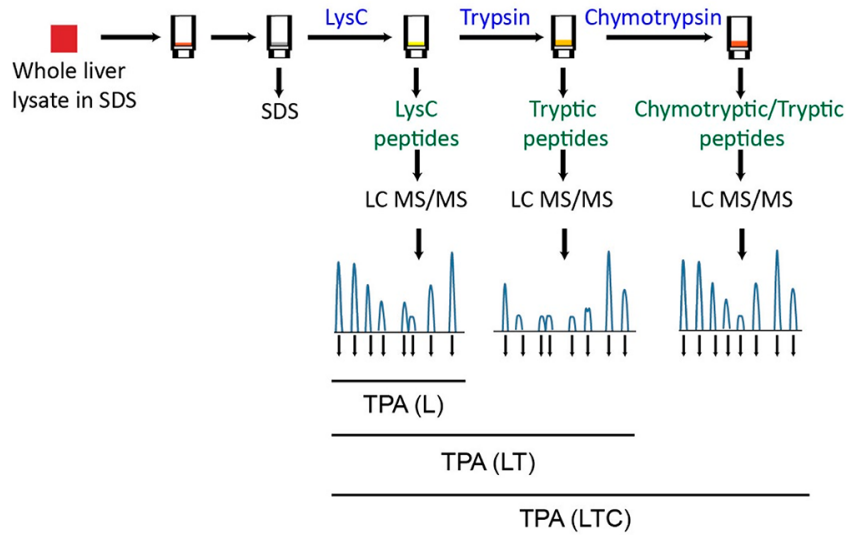

Figure 1. Experimental design. A. Whole liver SDS lysate was processed with FASP using successive digestion with LysC and trypsin. The resulting digests were spiked with stable isotope labeled (SIL) peptides and analyzed by LC-MS/MS. Proteins were quantified by the SIL-based approach and TPA. B. The liver lysate was processed with MED FASP using consecutive digestion with LysC (L), trypsin (T) and chymotrypsin (C). Each digest was analyzed in a separate LC-MS/MS run. Spectra from all digests were analyzed together by MaxQuant software and quantified by TPA.

\section{RESULTS}

\section{Experimental Design}

To compare the performance of the SIL-based method and the label- and standard free TPA method we analyzed protein concentrations in lysates of frozen liver biopsies from two donors, designated $\mathrm{A}$ and $\mathrm{B}$ (Figure 1). For a direct comparison of both methods, whole liver lysates were processed with FASP. To increase completeness of protein cleavage, proteins were successively digested with LysC and trypsin (Figure 1A). In contrast, to MED FASP protocol the digestion products were eluted together. After addition of the SIL-"heavy" peptide standards, the digests were analyzed in 95 min LC-MS/MS runs. In the SIL-based analysis, protein abundances were calculated from ratios of spectral intensity of "heavy" and "light" peptides. Global, TPA based, protein quantification was assessed using the raw intensities of all "light" peptides. In a parallel experiment, the liver lysates were processed by MED FASP using consecutive cleavage of proteins with LysC (L), trypsin (T), and chymotrypsin (C). (Figure 1B). Following LC-MS/MS analysis, specific protein concentrations were calculated by TPA.

\section{Concurrent SIL-Peptide Based and TPA Protein Quantification}

For analysis, 39 SIL-peptides that matched 35 unique target proteins were used. For 23 of these peptides, matching 23 proteins, the unlabeled counterparts were identified in at least three aliquots spiked with different amounts of SIL-standards, in at least one liver sample (Figure 2A) (SI Table 2). The 16 missing peptides were either not identified or the heavy form did not match the unlabeled counterpart peptide. Average variability in abundances of each of the 23 proteins between LC-MS/MS runs at various amount of spiked-in peptide, each analyzed in triplicate, was around 0.1 (CV, Figure 2B). Analysis of the "light" channel using the TPA method allowed quantification of about 2000 proteins, when considering only proteins identified with at least three peptides per single run (Table 1; SI Table 3). Out of these, between the LC-MS/MS runs, $27-30$ of the 35 "target" proteins were identified (Figure 2A, SI Table 4). The higher number of proteins quantified by
A

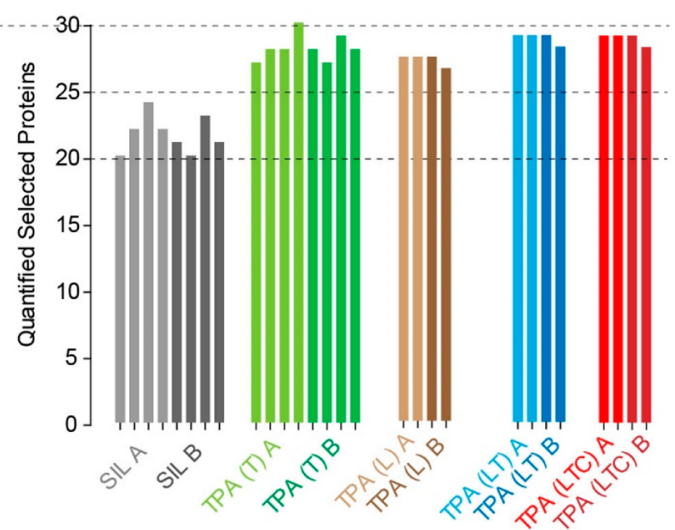

B

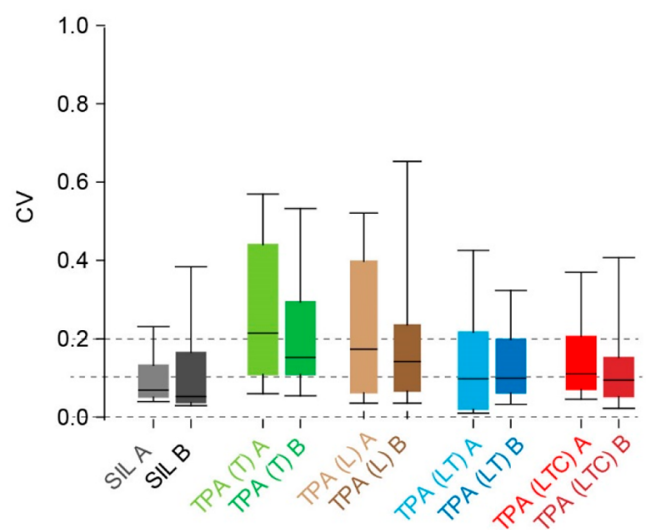

Figure 2. Comparison of quantification of the 34 selected proteins using stable isotope labeling (SIL) approach and TPA. Quantification of the proteins with TPA was assessed using "light channel" data from the SIL-based analysis (TPA (T)) or the three step MED FASP consecutive digestion using data from LC-MS/MS analysis of the LysC digests (TPA (L)), Lys C and tryptic digests (TPA (LT)), or LysC, tryptic and chymotryptic (TPA (LTC)). (A) Number of quantified proteins per single experiment (single bar). SIL and TPA(T) are averages from three LCMS/MS runs. (B) Coefficient of variation of concentrations for the quantified 34 proteins. 
Table 1. Identification of Peptides and Proteins Using Different Proteinases and Their Combinations

\begin{tabular}{|c|c|c|c|c|c|c|}
\hline \multirow[b]{2}{*}{ digestion strategy } & \multicolumn{3}{|c|}{ sample A } & \multicolumn{3}{|c|}{ sample B } \\
\hline & peptides & proteins & proteins with $\geq 3$ peptides & peptides & proteins & proteins with $\geq 3$ peptides \\
\hline LysC/trypsin ${ }^{a}$ & $19835 \pm 140$ & $3465 \pm 29$ & $2081 \pm 22$ & $18585 \pm 157$ & $3313 \pm 37$ & $1962 \pm 24$ \\
\hline LysC & $16786 \pm 455$ & $3624 \pm 65$ & $2046 \pm 46$ & $16370 \pm 47$ & $3582 \pm 1$ & $1983 \pm 20$ \\
\hline LysC+trypsin ${ }^{b}$ & $32400 \pm 805$ & $4916 \pm 57$ & $3150 \pm 60$ & $32042 \pm 335$ & $4930 \pm 5$ & $3106 \pm 19$ \\
\hline LysC+trypsin+Chymptrypsin ${ }^{3}$ & $38687 \pm 975$ & $5039 \pm 47$ & $3298 \pm 53$ & $38156 \pm 474$ & $5041 \pm 16$ & $3203 \pm 55$ \\
\hline
\end{tabular}

${ }^{a}$ Successive digestion (one LC-MS/MS run). ${ }^{b}$ consecutive digestion ( 2 or 3 LC-MS/MS runs). The values are biased by different data acquisition time and do not directly demonstrate advantages of multiple digestion. A direct comparison of the analytical depth, of data generated over the same acquisition time, is shown in Supplemental Table 8.
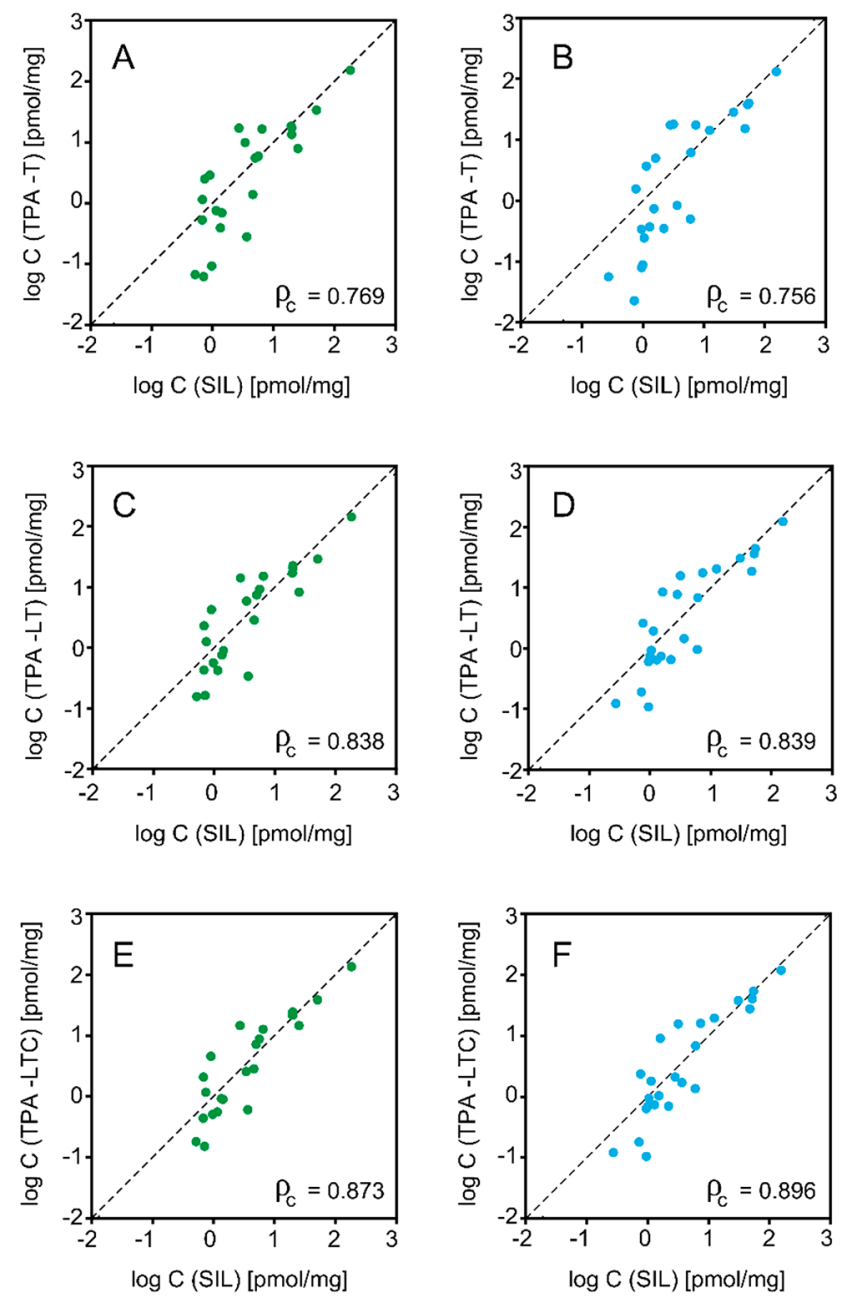

Figure 3. Correlations between concentrations of the selected proteins in liver $\mathrm{A}$ ( $\mathrm{A}, \mathrm{C}$, and $\mathrm{E})$ and liver $\mathrm{B}(\mathrm{B}, \mathrm{D}$, and $\mathrm{F})$. (A and B) correlation between SIL based and "light channel" TPA data of tryptic digests. (C and D) correlation between SIL based and TPA data from SIL and LysC and tryptic digests. (E and F) Correlation between SIL based and TPA data from SIL and LysC, tryptic and chymotryptic (TPA (LTC)). $\rho_{c}$, concordance correlation. ${ }^{36}$

TPA compared to the SIL-analysis reflects that some proteins were identified by several peptides but not by the selected "heavy"-"light" peptide pair. Comparison of the SIL- and TPA data showed that the former approach is more reproducible between single runs (Figure $2 \mathrm{~A}$ and $\mathrm{B}$ ). However, averaged protein concentrations from the triplicates from both approaches showed good correlations (0.783 and 0.870) (Figure $3 \mathrm{~A}$ and $\mathrm{B}$ ).
MED FASP Increases the Reproducibility and Improves Correlation with the SIL-Based Data

Accuracy of protein quantification in TPA relies on the depth of the proteomic analysis. This is not always achievable in a single and short LC-MS/MS run as the 95 min runs of tryptic peptides. As we have previously demonstrated, consecutive $^{17-19}$ or parallel $^{16,22}$ protein cleavages with several enzymes substantially increase the number of identified proteins and peptides per protein. Here, we conducted MED FASP based analyses using two or three consecutive cleavages (Figure 1B; SI Tables 5, 6, and 7). Compared to analyses of only tryptic peptides, searches of the combined runs of the LysC-trypsin (LT) or LysC-trypsin-chymotrypsin (LTC) digests doubled the number of identified peptides and increased the number of quantified proteins with more than $60 \%$ (Table 1). The MED FASP based analyses (LT and LTC) also increased reproducibility between experiments (Figure 2B). Whereas, average $\mathrm{CV}$ of protein titers assessed by analysis of single enzyme digests ( $\mathrm{L}$ or $\mathrm{T}$ ) are between 0.1 and 0.2 , the CVs from LT and LTC analyses were 0.1 and below, thus comparable to the variation observed for the SIL-based approach.

The MED FASP-TPA based quantitative data showed an improved correlation with the SIL-based measurements (Figure 2B and Figure 3). This reflects 2 and 2.4-fold increase of sequence coverage achieved by consecutive two or three digestion steps compared to single step protein cleavage (Figure 4). Also, the use of MED FASP for sample processing allows better peptide coverage of transmembrane proteins. Compared to single tryptic cleavages, digestion LT and LTC MED FASP-based analyses increased the content of proteins with predicted transmembrane domains (TMD) by 6 and $10 \%$, respectively (Table 2). For example, comparing only tryptic (T) with LTC digestion, the sequence coverages of the major uptake transporter SLCO1B1 increased from 11.9 to $21.8 \%$, respectively. Similarly, the coverage of the Multidrug Resistance Protein - the excretion transporter ABCB1 rose from 3.1 to $12 \%$ (Figure 4).

\section{DISCUSSION}

Protein quantification has never been an easy task in biology. It is therefore not surprising that this challenge persists in proteomics. Although it is a widespread belief that targeted analysis, using stable isotope peptide standards, is the proteomic method providing absolute and accurate values, recent studies revealed weaknesses of this analytical approach. ${ }^{23,24}$ It is now clear that protein abundance values generated by targeted proteomics are not absolute because they rely on many factors that are difficult to estimate or even out of control. Inaccuracies in determination of the amounts of 

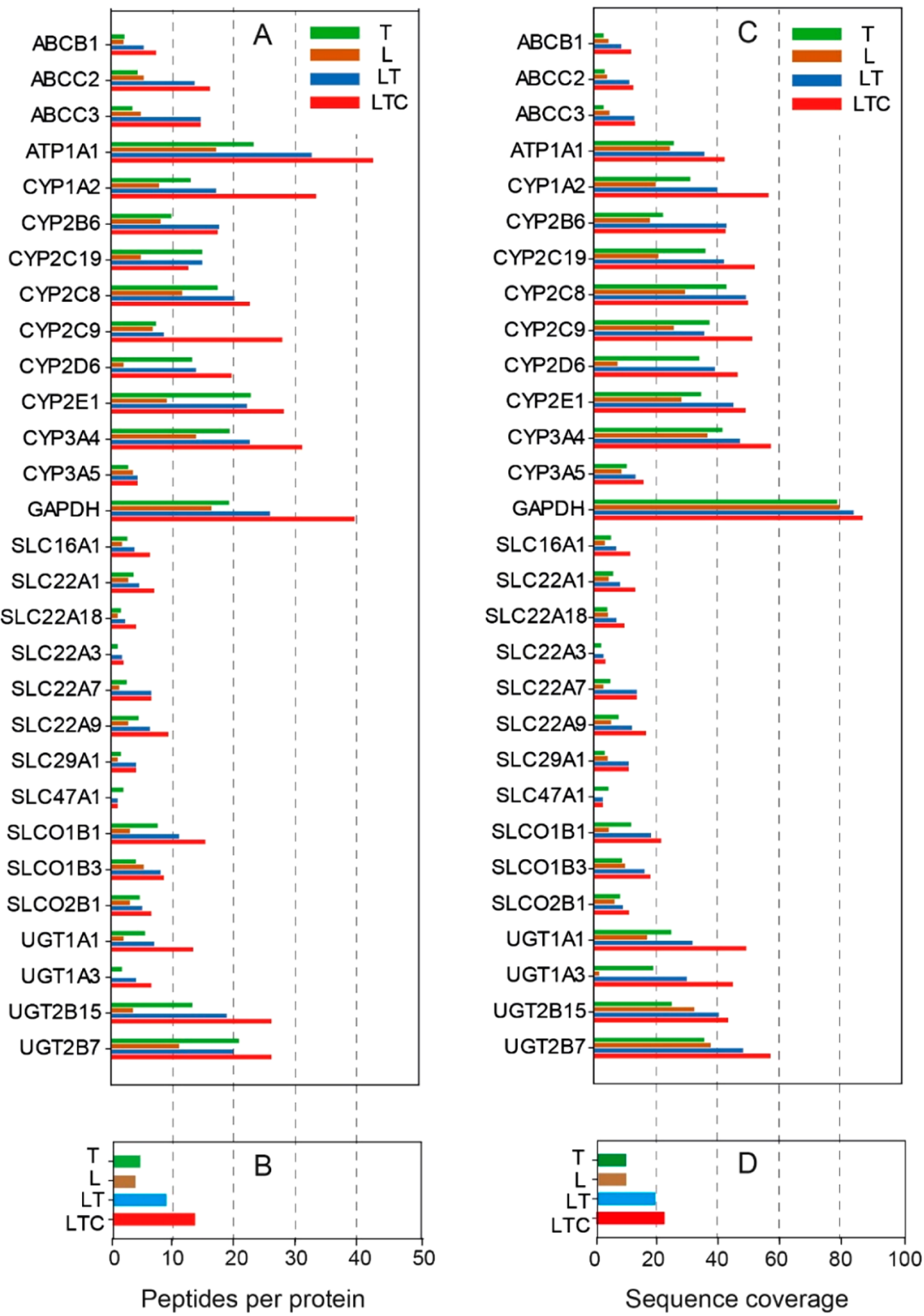

Figure 4. Number of peptides (A and B) and sequence coverage (C and D) of the target proteins. T, tryptic peptides identified in the "light channel" of the SIL-based analysis. L, LT, and LTC, peptides identified in the Lys C digest, LysC, and tryptic digests, and LysC, tryptic, and chymotryptic digests obtained by the MED FASP consecutive digestion, respectively. Panels B and D show median values of the data shown in the panels $\mathrm{A}$ and $\mathrm{C}$, respectively.

Table 2. Content of Proteins with Transmembrane Domains

\begin{tabular}{|c|c|c|c|}
\hline digestion strategy & $\begin{array}{l}\text { total proteins } \\
\text { identified }\end{array}$ & $\begin{array}{l}\text { with predicted } \\
\text { TMD }\end{array}$ & $\begin{array}{l}\% \\
\text { TMDs }\end{array}$ \\
\hline LysC/trypsin ${ }^{a}$ & 4536 & 738 & 16.3 \\
\hline LysC & 4169 & 620 & 14.9 \\
\hline LysC+trypsin ${ }^{b}$ & 5611 & 966 & 17.2 \\
\hline $\begin{array}{l}\text { LysC+trypsin } \\
\quad+\text { Chymptrypsin }\end{array}$ & 5735 & 1028 & 17.9 \\
\hline
\end{tabular}

${ }^{a}$ Successive digestion (one LC-MS/MS run). ${ }^{b}$ consecutive digestion ( 2 or 3 LC-MS/MS runs).

sample and the spiked-in standard, and uncertainty in the completeness in cleaving-out of the target peptide are key sample preparation features affecting the analyses. These factors are well-known and have been discussed in the past. ${ }^{25,26}$ To alleviate some of these technical problems, analysis of several target peptides for a single protein is recommended. In practice however, it is often difficult to find even one well behaving peptide. In this study we were able to quantify only 23 of 39 labeled peptides. In a much larger study by Kennedy et al., 512 standards were used but only 236 contributed to protein quantification. ${ }^{27}$ These difficulties can also be related to the sensitivity of the MS-analysis. To increase chances of detection of lower abundant proteins in the targeted assays and to bypass the limitation, analyses are often performed on isolated subcellular fractions and the measured values are corrected by scaling to the initial whole tissue sample. However, unexpected quantitative sample losses and limitations in subcellular tissue fractionation lead to values far away from accuracy. ${ }^{8,28}$ SRM analyses using full-length heavy labeled protein standards may provide more reliable protein quantification. ${ }^{29}$ However, it is not a general strategy because 
membrane proteins (more than $20 \%$ of the proteins in eukaryotic cells) are difficult to synthesize and handle.

Given this cloudy perspective of the targeted analyses, labeland standard-free analyses are powerful alternatives. In this work, we use TPA to analyze a group of key proteins involved in transport and metabolism of xenobiotics in human liver. We made advantage of the unique possibility to compare "a standard based method" using spike-in peptides with the "standard free" TPA. The data obtained in both ways show good to excellent correlation. In particular, data generated by consecutive digestion by the MED FASP strategy provided highly reproducible values of protein concentrations.

In the SIL-based analysis performed in this work, protein titers in the liver samples were assessed from ratios of $\mathrm{MS}^{1}$ signal abundances of labeled and nonlabeled peptides. This type of proteomic quantification can be considered as less reproducible and less sensitive compared to measurements by multiple or selected reaction monitoring (MRM or SRM) using isolated $\mathrm{MS}^{2}$ signals. Studies using the latter technology report for quantitative measurements coefficient variation values around $0.1^{27,30,31}$ and sensitivities down to few $\mathrm{fmol} /$ mg when target protein-enrichment strategies are used. ${ }^{32}$ However, in whole tissue lysates quantification of proteins with titers below $0.1 \mathrm{pmol} / \mathrm{mg}$ is rarely possible. ${ }^{27}$ Since our $\mathrm{MS}^{1}-$ SIL-based quantification share these properties, we consider it as a useful approach to validate TPA based quantification.

Targeted proteomics is probably the easiest way to quantify single or small groups of proteins in complex mixtures and appear to be well suited for fast and high-throughput measurements of protein titers for diagnostic purposes. Compared to this, global approaches are more timeconsuming. Neglecting these demands, the important advantage of the global approach compared to analyses using SILpeptide standards, is the ability to measure thousands of proteins in parallel in the sample as well as a possibility to reuse archival data sets generated in nonrelated scientific content. For example, the TPA analysis described herein allowed identification and quantification of, in addition to the targeted proteins, 243 additional proteins involved in absorption, distribution, metabolism, and excretion of drugs (SI Table 5).

As demonstrated with increased reproducibility after three consecutive digestion steps, the more data the data sets contain, the more accurate and reproducible the analysis will be. Of course, this is not only achievable by MED FASP and by other fractionation methods at the peptide level, but also by approaches involving very long columns and spectra acquisition times. ${ }^{33}$ Alternatively, MS-approaches generating more data, such as independent data acquisition, ${ }^{34}$ can further improve the accuracy in large-scale quantification of proteins. Notably, an important value of the TPA concept is that for protein quantification, $\mathrm{MS}^{1}$, as well as $\mathrm{MS}^{2}$ and $\mathrm{MS}^{3}$ outputs can be used in a TMT-multiplexed analysis. ${ }^{35}$

\section{CONCLUSION}

In this work, we demonstrate that consecutive protein digestion using MED FASP method with two or three proteinases improves the accuracy of TPA quantification to similar levels achievable by analysis using stable isotope labeled peptide standards. The presented data focus on quantification of transporters and enzymes metabolizing xenobiotics in liver. However, the conclusions drawn from this study seems to be general and thus applicable to other groups of proteins.

\section{ASSOCIATED CONTENT}

\section{Supporting Information}

The Supporting Information is available free of charge on the ACS Publications website at DOI: 10.1021/acs.jproteome.8b00549.

(PDF)

Supplementary Table 1 . Stable isotope labeled peptides used in the analysis. Supplementary Table 2. SIL-based quantification of target proteins. Supplementary Table 3. TPA based global quantification of proteins using the "light" channel of the SIL-based analysis. Supplementary Table 4. Summary of SIL- and TPA based analyses of target protein. Supplementary Table 5. TPA based global quantification of proteins processed by MED FASP using 3 enzymes (LysC, trypsin and chymotrypsin). Supplementary Table 6. TPA based global quantification of proteins processed by MED FASP using two enzymes (LysC and trypsin). Supplementary Table 7. TPA based global quantification of proteins digested using LysC. Supplementary Table 8. Comparison of peptide and protein identifications by analysis of samples prepared using single tryptic digestion and consecutive digestion with three enzymes. For both types of analysis, the same mass spectrometry acquisition time was used (XLSX)

\section{AUTHOR INFORMATION}

\section{Corresponding Author}

*Phone: +49 898578 2205. Fax: +49 898578 2219. E-mail: jwisniew@biochem.mpg.de.

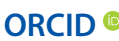

Jacek R Wiśniewski: 0000-0002-8452-5095 Christine Wegler: 0000-0002-2810-7518

Notes

The authors declare no competing financial interest.

\section{ACKNOWLEDGMENTS}

We are grateful to Prof. Matthias Mann for continuous support. We thank Katharina Zettl for technical support. The work was funded by Max-Planck Society for the Advancement of Science by the German Research Foundation (DFG/ Gottfried Wilhelm Leibniz Prize), and the Swedish Research Council (approval number 1951 and 5715).

\section{REFERENCES}

(1) Wisniewski, J. R.; Ostasiewicz, P.; Dus, K.; Zielinska, D. F.; Gnad, F.; Mann, M. Extensive quantitative remodeling of the proteome between normal colon tissue and adenocarcinoma. Mol. Syst. Biol. 2012, 8, 611.

(2) Wisniewski, J. R.; Rakus, D. Multi-enzyme digestion FASP and the 'Total Protein Approach'-based absolute quantification of the Escherichia coli proteome. J. Proteomics 2014, 109C, 322-331.

(3) Wisniewski, J. R. Label-Free and Standard-Free Absolute Quantitative Proteomics Using the "Total Protein" and "Proteomic Ruler" Approaches. Methods Enzymol. 2017, 585, 49-60.

(4) Wisniewski, J. R.; Hein, M. Y.; Cox, J.; Mann, M. A 'proteomic ruler' for protein copy number and concentration estimation without spike-in standards. Mol. Cell. Proteomics 2014, 13, 3497-3506.

(5) Wisniewski, J. R.; Gizak, A.; Rakus, D. Integrating Proteomics and Enzyme Kinetics Reveals Tissue-Specific Types of the Glycolytic and Gluconeogenic Pathways. J. Proteome Res. 2015, 14, 3263-3273. 
(6) Wisniewski, J. R.; Friedrich, A.; Keller, T.; Mann, M.; Koepsell, $\mathrm{H}$. The Impact of High-Fat Diet on Metabolism and Immune Defense in Small Intestine Mucosa. J. Proteome Res. 2015, 14, 353-365.

(7) Wisniewski, J. R.; Vildhede, A.; Noren, A.; Artursson, P. In-depth quantitative analysis and comparison of the human hepatocyte and hepatoma cell line HepG2 proteomes. J. Proteomics 2016, 136, 234247.

(8) Wisniewski, J. R.; Wegler, C.; Artursson, P. Subcellular fractionation of human liver reveals limits in global proteomic quantification from isolated fractions. Anal. Biochem. 2016, 509, 8288

(9) Wisniewski, J. R.; Dus-Szachniewicz, K.; Ostasiewicz, P.; Ziolkowski, P.; Rakus, D.; Mann, M. Absolute Proteome Analysis of Colorectal Mucosa, Adenoma, and Cancer Reveals Drastic Changes in Fatty Acid Metabolism and Plasma Membrane Transporters. J. Proteome Res. 2015, 14 (9), 4005-18.

(10) Doll, S.; Dressen, M.; Geyer, P. E.; Itzhak, D. N.; Braun, C.; Doppler, S. A.; Meier, F.; Deutsch, M. A.; Lahm, H.; Lange, R.; Krane, M.; Mann, M. Region and cell-type resolved quantitative proteomic map of the human heart. Nat. Commun. 2017, 8 (1), 1469.

(11) Morgenstern, M.; Stiller, S. B.; Lubbert, P.; Peikert, C. D.; Dannenmaier, S.; Drepper, F.; Weill, U.; Hoss, P.; Feuerstein, R.; Gebert, M.; Bohnert, M.; van der Laan, M.; Schuldiner, M.; Schutze, C.; Oeljeklaus, S.; Pfanner, N.; Wiedemann, N.; Warscheid, B. Definition of a High-Confidence Mitochondrial Proteome at Quantitative Scale. Cell Rep. 2017, 19 (13), 2836-2852.

(12) Rieckmann, J. C.; Geiger, R.; Hornburg, D.; Wolf, T.; Kveler, K.; Jarrossay, D.; Sallusto, F.; Shen-Orr, S. S.; Lanzavecchia, A.; Mann, M.; Meissner, F. Social network architecture of human immune cells unveiled by quantitative proteomics. Nat. Immunol. 2017, 18 (5), 583-593.

(13) Gautier, E. F.; Ducamp, S.; Leduc, M.; Salnot, V.; Guillonneau, F.; Dussiot, M.; Hale, J.; Giarratana, M. C.; Raimbault, A.; Douay, L.; Lacombe, C.; Mohandas, N.; Verdier, F.; Zermati, Y.; Mayeux, P. Comprehensive Proteomic Analysis of Human Erythropoiesis. Cell Rep. 2016, 16 (5), 1470-1484.

(14) Groer, C.; Bruck, S.; Lai, Y.; Paulick, A.; Busemann, A.; Heidecke, C. D.; Siegmund, W.; Oswald, S. LC-MS/MS-based quantification of clinically relevant intestinal uptake and efflux transporter proteins. J. Pharm. Biomed. Anal. 2013, 85, 253-61.

(15) Wisniewski, J. R.; Gaugaz, F. Z. Fast and sensitive total protein and Peptide assays for proteomic analysis. Anal. Chem. 2015, 87 (8), 4110-6.

(16) Wisniewski, J. R.; Zougman, A.; Nagaraj, N.; Mann, M. Universal sample preparation method for proteome analysis. Nat. Methods 2009, 6 (5), 359-62.

(17) Wisniewski, J. R.; Mann, M. Consecutive proteolytic digestion in an enzyme reactor increases depth of proteomic and phosphoproteomic analysis. Anal. Chem. 2012, 84 (6), 2631-7.

(18) Wisniewski, J. R. Quantitative Evaluation of Filter Aided Sample Preparation (FASP) and Multienzyme Digestion FASP Protocols. Anal. Chem. 2016, 88 (10), 5438-43.

(19) Stachowicz, A.; Siudut, J.; Suski, M.; Olszanecki, R.; Korbut, R.; Undas, A.; Wisniewski, J. R. Optimization of quantitative proteomic analysis of clots generated from plasma of patients with venous thromboembolism. Clin. Proteomics 2017, 14, 38.

(20) Vizcaino, J. A.; Deutsch, E. W.; Wang, R.; Csordas, A.; Reisinger, F.; Rios, D.; Dianes, J. A.; Sun, Z.; Farrah, T.; Bandeira, N.; Binz, P. A.; Xenarios, I.; Eisenacher, M.; Mayer, G.; Gatto, L.; Campos, A.; Chalkley, R. J.; Kraus, H. J.; Albar, J. P.; MartinezBartolome, S.; Apweiler, R.; Omenn, G. S.; Martens, L.; Jones, A. R.; Hermjakob, H. ProteomeXchange provides globally coordinated proteomics data submission and dissemination. Nat. Biotechnol. 2014, 32 (3), 223-6.

(21) Wisniewski, J. R.; Rakus, D. Multi-enzyme digestion FASP and the 'Total Protein Approach'-based absolute quantification of the Escherichia coli proteome. J. Proteomics 2014, 109, 322-31.

(22) Bryk, A. H.; Wisniewski, J. R. Quantitative Analysis of Human Red Blood Cell Proteome. J. Proteome Res. 2017, 16 (8), 2752-2761.
(23) Wegler, C.; Gaugaz, F. Z.; Andersson, T. B.; Wisniewski, J. R.; Busch, D.; Groer, C.; Oswald, S.; Noren, A.; Weiss, F.; Hammer, H. S.; Joos, T. O.; Poetz, O.; Achour, B.; Rostami-Hodjegan, A.; van de Steeg, E.; Wortelboer, H. M.; Artursson, P. Variability in Mass Spectrometry-based Quantification of Clinically Relevant Drug Transporters and Drug Metabolizing Enzymes. Mol. Pharmaceutics 2017, 14 (9), 3142-3151.

(24) Achour, B.; Dantonio, A.; Niosi, M.; Novak, J. J.; Fallon, J. K.; Barber, J.; Smith, P. C.; Rostami-Hodjegan, A.; Goosen, T. C. Quantitative Characterization of Major Hepatic UDP-Glucuronosyltransferase Enzymes in Human Liver Microsomes: Comparison of Two Proteomic Methods and Correlation with Catalytic Activity. Drug Metab. Dispos. 2017, 45 (10), 1102-1112.

(25) Carr, S. A.; Abbatiello, S. E.; Ackermann, B. L.; Borchers, C.; Domon, B.; Deutsch, E. W.; Grant, R. P.; Hoofnagle, A. N.; Huttenhain, R.; Koomen, J. M.; Liebler, D. C.; Liu, T.; MacLean, B.; Mani, D. R.; Mansfield, E.; Neubert, H.; Paulovich, A. G.; Reiter, L.; Vitek, O.; Aebersold, R.; Anderson, L.; Bethem, R.; Blonder, J.; Boja, E.; Botelho, J.; Boyne, M.; Bradshaw, R. A.; Burlingame, A. L.; Chan, D.; Keshishian, H.; Kuhn, E.; Kinsinger, C.; Lee, J. S.; Lee, S. W.; Moritz, R.; Oses-Prieto, J.; Rifai, N.; Ritchie, J.; Rodriguez, H.; Srinivas, P. R.; Townsend, R. R.; Van Eyk, J.; Whiteley, G.; Wiita, A.; Weintraub, S. Targeted peptide measurements in biology and medicine: best practices for mass spectrometry-based assay development using a fit-for-purpose approach. Mol. Cell. Proteomics 2014, 13 (3), 907-17.

(26) Trotschel, C.; Poetsch, A. Current approaches and challenges in targeted absolute quantification of membrane proteins. Proteomics 2015, 15 (5-6), 915-29.

(27) Kennedy, J. J.; Whiteaker, J. R.; Schoenherr, R. M.; Yan, P.; Allison, K.; Shipley, M.; Lerch, M.; Hoofnagle, A. N.; Baird, G. S.; Paulovich, A. G. Optimized Protocol for Quantitative Multiple Reaction Monitoring-Based Proteomic Analysis of Formalin-Fixed, Paraffin-Embedded Tissues. J. Proteome Res. 2016, 15 (8), 2717-28.

(28) Harwood, M. D.; Russell, M. R.; Neuhoff, S.; Warhurst, G.; Rostami-Hodjegan, A. Lost in centrifugation: accounting for transporter protein losses in quantitative targeted absolute proteomics. Drug Metab. Dispos. 2014, 42 (10), 1766-72.

(29) Simicevic, J.; Schmid, A. W.; Gilardoni, P. A.; Zoller, B.; Raghav, S. K.; Krier, I.; Gubelmann, C.; Lisacek, F.; Naef, F.; Moniatte, M.; Deplancke, B. Absolute quantification of transcription factors during cellular differentiation using multiplexed targeted proteomics. Nat. Methods 2013, 10 (6), 570-6.

(30) Abbatiello, S. E.; Mani, D. R.; Schilling, B.; Maclean, B.; Zimmerman, L. J.; Feng, X.; Cusack, M. P.; Sedransk, N.; Hall, S. C.; Addona, T.; Allen, S.; Dodder, N. G.; Ghosh, M.; Held, J. M.; Hedrick, V.; Inerowicz, H. D.; Jackson, A.; Keshishian, H.; Kim, J. W.; Lyssand, J. S.; Riley, C. P.; Rudnick, P.; Sadowski, P.; Shaddox, K.; Smith, D.; Tomazela, D.; Wahlander, A.; Waldemarson, S.; Whitwell, C. A.; You, J.; Zhang, S.; Kinsinger, C. R.; Mesri, M.; Rodriguez, H.; Borchers, C. H.; Buck, C.; Fisher, S. J.; Gibson, B. W.; Liebler, D.; Maccoss, M.; Neubert, T. A.; Paulovich, A.; Regnier, F.; Skates, S. J.; Tempst, P.; Wang, M.; Carr, S. A. Design, implementation and multisite evaluation of a system suitability protocol for the quantitative assessment of instrument performance in liquid chromatography-multiple reaction monitoring-MS (LC-MRM-MS). Mol. Cell. Proteomics 2013, 12 (9), 2623-39.

(31) Zhang, H.; Liu, Q.; Zimmerman, L. J.; Ham, A. J.; Slebos, R. J.; Rahman, J.; Kikuchi, T.; Massion, P. P.; Carbone, D. P.; Billheimer, D.; Liebler, D. C. Methods for peptide and protein quantitation by liquid chromatography-multiple reaction monitoring mass spectrometry. Mol. Cell. Proteomics 2011, 10 (6), M110 006593.

(32) Keshishian, H.; Addona, T.; Burgess, M.; Kuhn, E.; Carr, S. A Quantitative, multiplexed assays for low abundance proteins in plasma by targeted mass spectrometry and stable isotope dilution. Mol. Cell. Proteomics 2007, 6 (12), 2212-29.

(33) Horie, K.; Kamakura, T.; Ikegami, T.; Wakabayashi, M.; Kato, T.; Tanaka, N.; Ishihama, Y. Hydrophilic interaction chromatography 
using a meter-scale monolithic silica capillary column for proteomics LC-MS. Anal. Chem. 2014, 86 (8), 3817-24.

(34) Gillet, L. C.; Navarro, P.; Tate, S.; Rost, H.; Selevsek, N.; Reiter, L.; Bonner, R.; Aebersold, R. Targeted data extraction of the MS/MS spectra generated by data-independent acquisition: a new concept for consistent and accurate proteome analysis. Mol. Cell. Proteomics 2012, 11 (6), O111 016717.

(35) Vildhede, A.; Nguyen, C.; Erickson, B. K.; Kunz, R. C.; Jones, R.; Kimoto, E.; Bourbonais, F.; Rodrigues, A. D.; Varma, M. V. Comparison of proteomic quantification approaches for hepatic drug transporters: multiplexed global quantitation correlates with targeted proteomic quantitation. Drug Metab. Dispos. 2018, 46, 692-696.

(36) Lin, L. I. A concordance correlation coefficient to evaluate reproducibility. Biometrics 1989, 45 (1), 255-68. 